\title{
Adaptation of First-Year Architectural Design Studio Resources Through Accessibility
}

\author{
Derya Uzal \\ MEF University, Faculty of Arts, Design and Architecture, İstanbul Turkey, \\ Basak Eren \\ MEF University, Faculty of Arts, Design and Architecture, İstanbul Turkey,
}

Received: May 10th 2021, Revised: June 19tt 2021, Accepted: June 22nd 2021.

Refer: Uzal, D., Eren, B., (2021), Adaptation of First-Year Architectural Design Studio Resources Through Accessibility, Journal of Design Studio, V.3, N.1, pp 125-135,

D. Uzal ORCID: 0000-0002-1516-592X , B. Eren ORCID: 0000-0003-4680-5886

DOI: $10.46474 /$ jds. 935436 https://doi.org/10.46474/jds.935436

\begin{abstract}
This paper aims to discuss possible adaptations of the essential resources for the first-year architectural design studio's second term under COVID-19 lockdown regulations through experiences from MEF University First-Year Design Studio. Design Studio fundamentals, such as accessibility and materiality, needed to be adapted to studio participants' changing opportunities and places. The second term of the first-year design studio at MEF University is built upon the basic knowledge gained from the first term by improving its physical aspects such as structure, material, and site by forming direct relationships with the resources through analysis and experimentation. Its adaptation to remote studio poses significant difficulties with its intense tactile and material state. New resources and adaptations to the remote studio are grouped under three categories: Curriculum, studio as a workspace, and site. Remote studio experiences are analyzed through changing resources to uncover new possible achievements. Even though there are still irreplaceable components of the regular studio structure, the paper searches for possible adaptations to overcome these challenges of architectural design studio during remote teaching by reassessment of the resources with the accessibility theme.
\end{abstract}

Keywords: First-year education, Remote studio, Architectural design studio, Accessible resources

\section{Introduction}

In March 2020 with the declaration of COVID19 as a pandemic, design education had reached a significant crossroad with the obligation of leaving the studio space as one of the most critical components and continuing education remotely. A common studio ground has extra importance for first-year design courses to meet with other students and learn collectively. Studio space contributes to the projects by enabling physical experience, temporal and cultural immersion, specific use of material space, and learning-by-doing (Corazzo, 2019). It plays a crucial role in providing a basis for essentials of first-year education with collective productions, excursions, and experimentations.
The second term of the year differs from the first with more complex material, and siterelated problems. During the second term, the need for studio space and related infrastructures increases. It becomes a critical phase of MEF University Faculty of Arts, Design, and Architecture (FADA) first-year education to meet all the learnings from the first term with additional spatial and material engagements in design projects. In recent years, projects aimed to introduce complex topographical and structural relationships with architecture and interior design students through collective site visits, material and structure experiments (Fig.1). The project objectives held holistically with consecutive empirical assignments such as 


\section{Journal of \\ Design Studio}

v:3 n:1 July 2021

material experiments, structural studies, and site analysis. However, losing the common theme and formed the new agenda of the remote studio. studio ground with pandemic restrictions
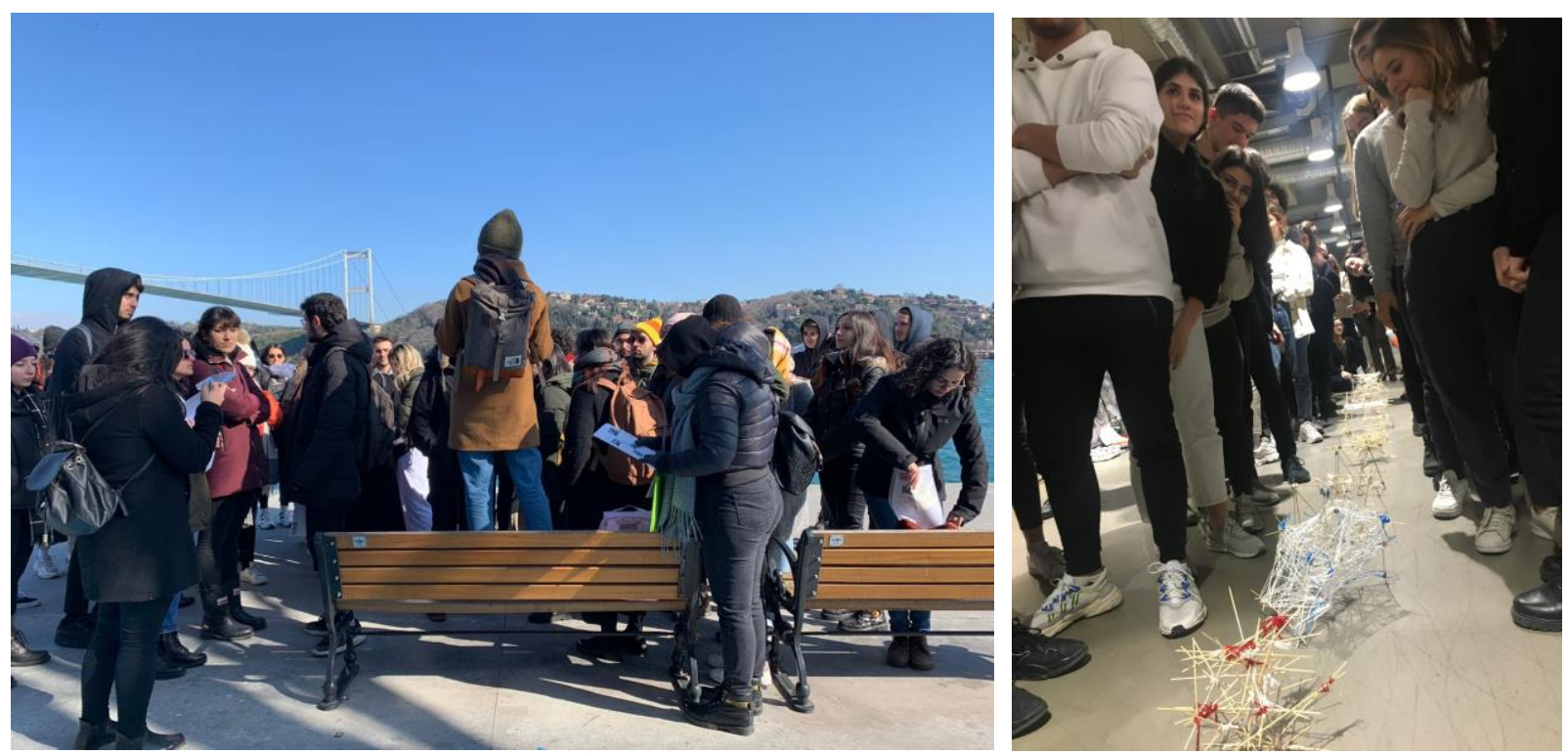

Figure 1.a: Collective site visit to Bebek, İstanbul for 'Amphibian' project, 2020 Spring

Figure 1.b: Structure workshop 'Bear-er' to carry maximum load possible, 2020 Spring

precluded these collective studies and brought the need to adapt it to the changing contexts of each individual.

In addition to first-year's significant spatial bonds, MEF FADA design studios - HANGAR plays a crucial role in providing all the infrastructure for design education and hosting various instructor, designer, and student encounters (Fig.2). Studio space acts as the facilitator of the design courses. While the unifying power of the studio space played a key role in first-year education, its absence causes reconsidering the whole term structure with transferring offerings of the physical space to other resources. Since the existing term structure is highly connected to the common studio context, it was inevitable to produce a new adaptation of those resources -especially site-related- to accomplish the same set of goals and rebuild the rich atmosphere of the studio with diverse encounters, experimentations, and collective productions. Considering the reasons above, the studio's resources, which constitutes its integrity, reconsidered with the accessibility

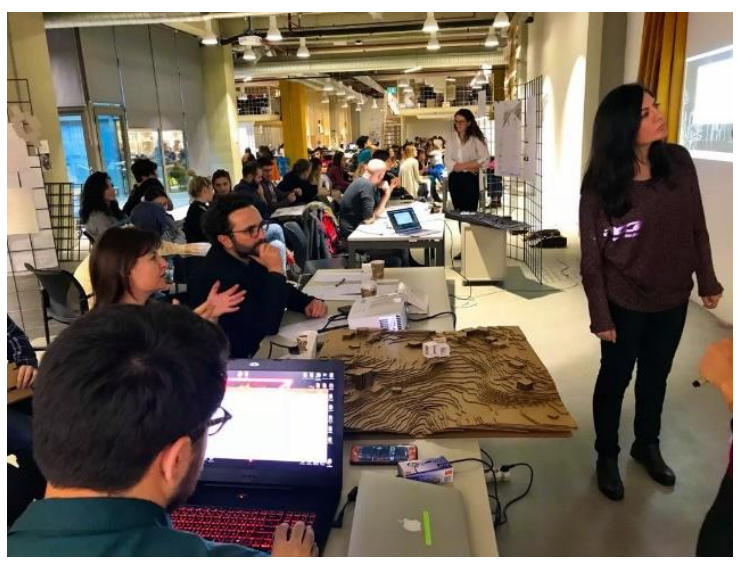

Figure 2. An open-jury session in MEF FADA Hangar Studio. Photo: Ipek Yürekli

\section{Methods}

In design education, the studio defines not only the physical space of learning; but also a working culture, mode of teaching and learning, and program of activity (Schön, 1987). Consequently, studio space acts as a unifying space of all in the process. In pre-covid terms, the studio is based on the resources offered by the common studio space or collectively experimented at the site by excursions. 


\section{Journal of \\ Design Studio}

v:3 n:1 July 2021

Replacement of the studio with online mediums and changing collective experiences with individual, various sites result in adaptations of the used resources and their formulation. Several resources contribute to the development of the projects. Some of them needed to be changed in adaptation to the remote studio and new ones were added. They are analyzed under three main categories according to their engagements: (1) curriculum, (2) studio space as a workspace, and (3) site. Under these categories, the paper aims to analyze reflections of these adaptations to the student projects and track their changing effects in projects. Remote studio projects will be analyzed through resources to uncover new possible achievements in research, analytical expressions, advanced spatial thinking, and drawing phases of the projects.

\section{Curriculum}

The curriculum plays a critical guiding role in the first-year architectural design studio where students are not well-equipped to guide themselves to reach basic proficiency to undertake a design project. Projects are built step by step onto each other to reach a set of skills and develop a design perspective. In the previous years, the term was formed by three consecutive projects, each exploring material and structural natures of architecture first separately in detail and then together with combining all learnings of the semester on a collectively explored site around a specific theme.

Despite all the disadvantages of the remote studio with losing some crucial resources, the main structure of the second term curriculum is redefined to achieve a similar level of structural, material, and spatial competencies. While fixing the curriculum outcomes for the online studio, some questions arise: Which tools did students have had before? Moreover, which circumstances offered by the curriculum encouraged students to explore these tools? Besides the visible curriculum, the interwoven nature of the physical studio creates an unplanned, natural, and inevitable 'hidden curriculum'. The hidden curriculum supports the visible curriculum in the natural flow of the studio with its rich learning environment, casual encounters, and collective studies. Without the studio, the hidden curriculum needed to be exposed and included to the visible one to recreate the studio atmosphere. Instructors are prompted to include the casual interactions of the physical studio intentionally within each project to create a more dynamic studio structure to reach the desired level of proficiency. The curriculum becomes a more layered and planned element guiding the studio.

The curriculum is revised as two projects combining all instead of three to create the gaps to insert dynamic modules without abandoning curriculum objectives: (1) Hydrophilia exploring material nature first from tactile experiments, then from remote analysis of the sites around the world, (2) Perform-X focusing on the adaptation of the given theme to local with site analysis and local information. 'Dynamic' structure of the studio is planned first with creating gaps between regular studio days to insert new workshop modules to introduce alternative topics to recreate rich encounters of the studio during the term intentionally. Second, limited screen time and interaction of students increased through the online logbooks, including their all productions to simulate the productive environment of the studio.

Firstly, in order to break the studio routine, Workshop modules by experts around the world are included in the curriculum. The studios had become monotonous due to the limited interaction provided by the static grid view of Zoom with three studio meetings a week. To break this monotonous flow and motivate the students, the introduction of diverse meetings became critical. In addition to dynamizing the static studio structure, workshops aimed to stage new encounters with the experts deliberately, which was the spontaneous activity in the studio space in pre-covid terms. Every one of two Wednesday projects are planned as workshop days; the studio hosted seven workshops in 14 weeks by experts of disciplines other than architecture. Guests are asked for lectures and daily workshops on their field of expertise under the theme of "accessible 


\section{Journal of \\ Design Studio \\ v:3 n:1 July 2021}

resources". Scheduled workshops aimed to introduce coincidental dynamic encounters and their learnings existing in the studio's nature by embedding them into the curriculum. Moreover, meeting with guest experts expands the architectural curriculum by merging it across disciplines (Fig.3). As a result, the guests' variety was more incredible than ever with increasing accessibility to the world virtually.

Workshops reveal the feeling of togetherness through these intermediaries and create an inviting and exposed atmosphere. Workshops have challenged students to design and produce in a limited time, exhibit their work on online platforms, and get feedback from experts. Furthermore, they inspired students to develop a new perspective through everyday objects around their usual desks, rooms, or houses as designers.
Secondly, Logbooks as interactive sketchbooks by students are introduced to flex studio hours and expand to a timeless medium for increasing interaction between the studio participants and their works to create the virtual studio environment. Logbooks are Google Slides documents for students to document their project processes in detail which are open to the entire studio, which is an expanded portfolio rather than an archive, allowing the instructors to give feedback and follow students' productions (Fig.4). In previous terms, instructors were involved in the students' processes from their studio desks with seeing previous models and sketchbooks, resulting in comprehensive perception due to the physical environment. In the absence of studio space, logbooks became vital media for both students and instructors in order to manage the processbased nature of the architectural design studio.
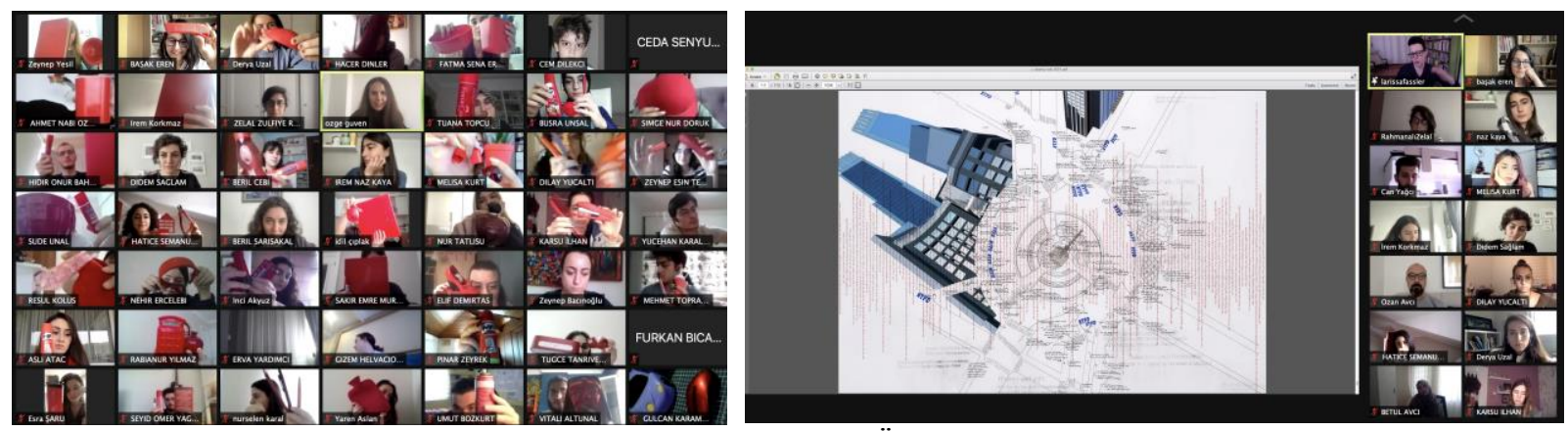

Figure 3.a: Workshop Wednesdays \#7: tasarl(m) workshop by Özge Güven, April 28th 2021

Figure 3.b: Workshop Wednesdays \#5: urban fabric, social fabric workshop by Larissa Fassler, April 14th 2021
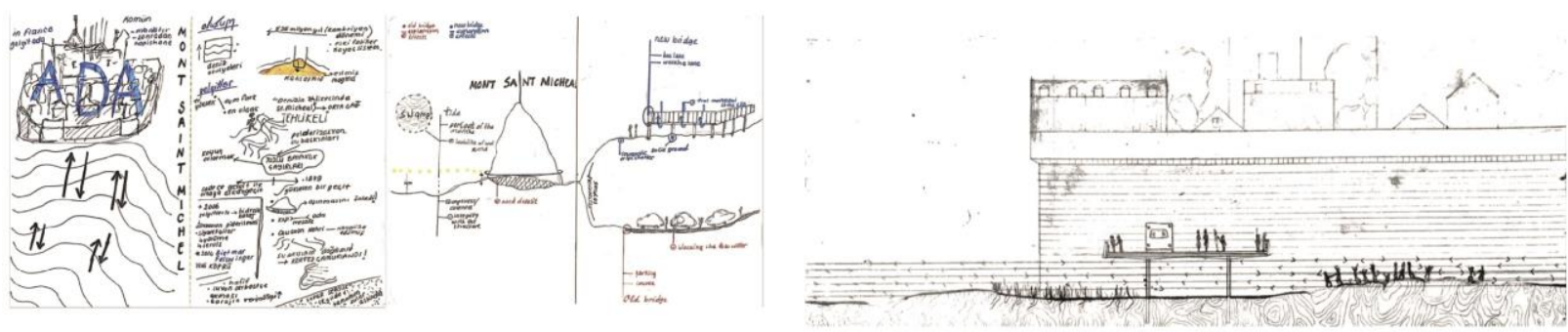

Figure 4: Pages from a Logbook: site research and drawings. Project: Ekin Nakip 


\section{Journal of \\ Design Studio}

v:3 n:1 July 2021

As a result of the changes in the curriculum, the studio became a more structured environment to include diverse encounters deliberately. Logbooks replaced sketchbooks and studio workspaces, also used as accessible resources to create the online studio environment where students interact with each other's works. Especially for the first-year students who are new to the field, it is crucial to see more works to inspire and motivate students. Decreasing visibility and increasing isolation of the students reduced with online timeless existence and exhibition of students.

\section{Studio Space}

The studio and campus space was undertaking the role of activating the static structure of the studio and students in regular terms. It also increases the diversity of the design discussions with different disciplines and individuals with the hidden curriculum. Pandemic restrictions precluded these possible encounters. We have experienced the importance of MEF studios, HANGAR to complement education and build a community over time. HANGAR is a 24-hour open learning place open to the personalizations of the students where multiplications of the learning channels are encouraged with its open structure. After the accessibility of this productive environment was restricted, its infrastructures needed to be transferred to virtual. Interaction between students, personalized working environments, exhibitions, laboratory spaces, equipment, materials, forums, and events are translated to Zoom environment and home resources.

The first adaptation was changing the studio structure to increase the limited interaction level of the virtual meetings since Zoom meetings remained incapable of motivating students with the pre-covid studio structure. Group structures are changed for activating students and increase even involuntary interactions. The usual group structure of the first-year studio, as approximately 13 students and one instructor paired with another group 28 people, created a more prosperous studio production, resulting in more fulfilling feedback from both instructors and fellow students.
MEF design studios have never been a hierarchical space with authoritative instructor figures; the studio acts as a collective production and discussion space with its horizontal organization. Digital medium's nature spotlighting the speaker and putting them to the center of the studio poses the risk of making instructors the avoided authority figure. The first-year students tend to seek a leading figure showing directions, trues and falses; which is an essential discussion in the design studios. The paired instructor and group system was beneficial in challenging that natural authoritative position of virtual meeting interfaces with changing the singular position of instructor, encouraging discussions, and introducing different ideas that are in the design studio's nature. As a result, all students are encouraged to raise their voices and become active players of the Zoom grid.

The second adaptation was replacing the material resources and equipment, forming the framework of the project from site and studiospecific to accessible ones. In pre-covid terms, the collective experimentation and discussions in the studio were replaced by material experiments conducted from home and with domestic resources. In previous years, physical experiments, such as building collective site models or load-bearing tests for a structure, resulted in efficient group work, also reinforced student relationships and studio culture. Similar but more primitive experiments based on accessible materials from home are also discussed on interactive whiteboards (Fig.5). Considered as alternative processes on materials and equipment provided by the studio's lab, WWs allowed a wide variety of materials to be tested. Although material diversity was achieved, the inherent efficiency, level of detail, and student interaction were not as strong as face-to-face studios. Nevertheless, the collaborative work environment is considered as an opportunity to recall this material richness into the studio. 


\section{Journal of}

\section{Design Studio}

v:3 n:1 July 2021

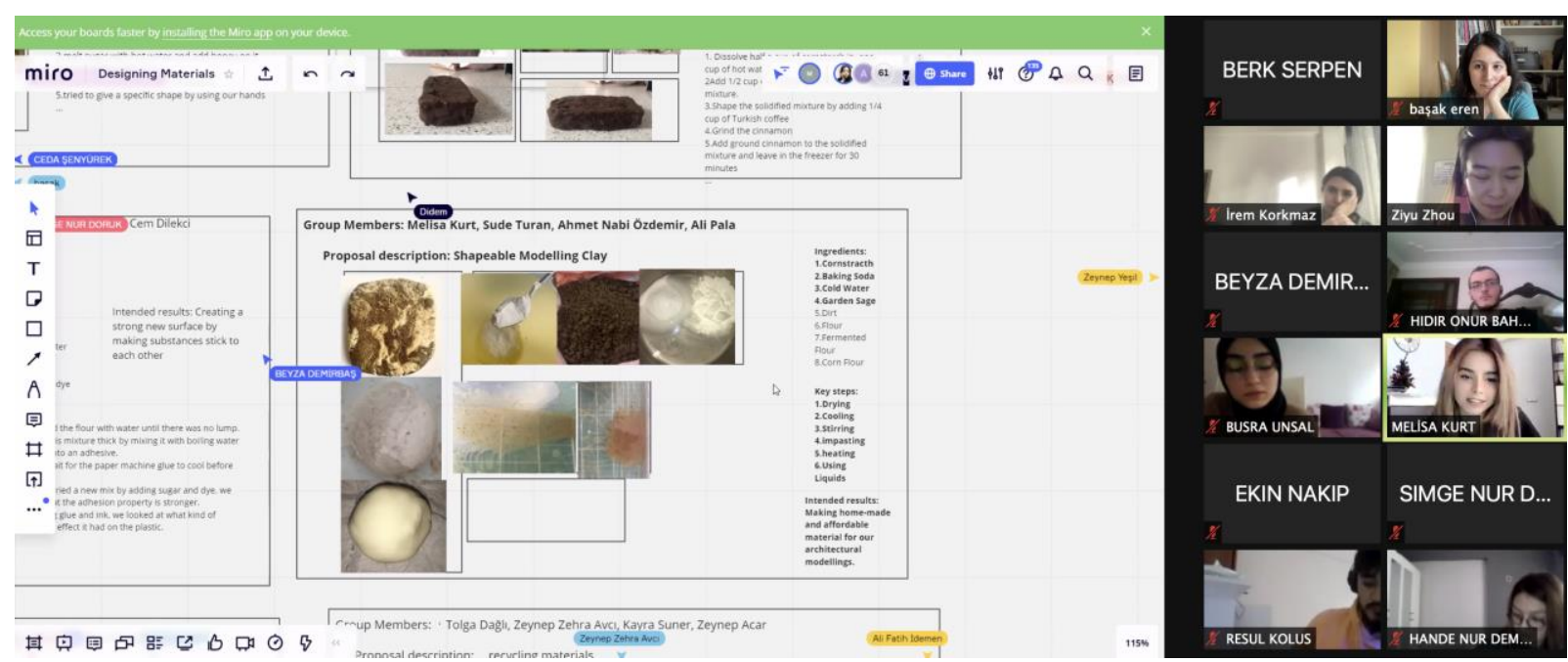

Figure 5: Collaborative whiteboard studies for Wednesday Workshop \#2: designing materials by Ziyu Zhou, March 3rd, 2021
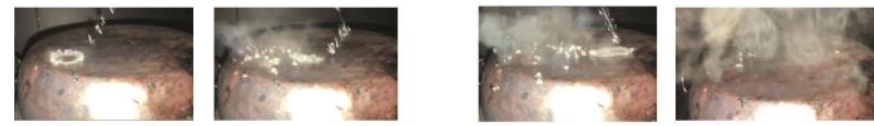

Dancing of water on
the surface thanks

Initial contact of
water with a surface higher than

surface higher than
its boiling point
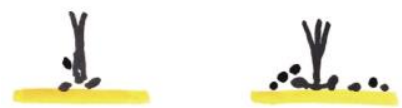
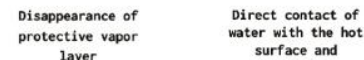

water with the hot
surface and

surface and
evaporation

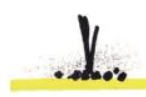

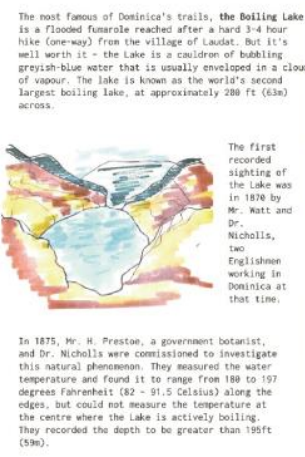
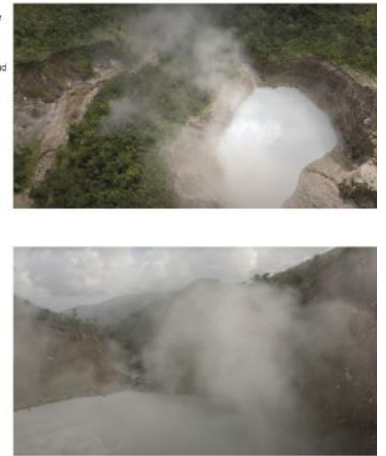

Figure 6: Water-based experiment diagram and site research. Project: Seyid Ömer Yağmurcu

During the regular term, the first project was designed as a process construction on an assigned topography at micro and macro scales with models and drawings. School's model laboratory, equipment, and various materials by the rich stationery enabled numerous material experiments and made the project effective. Without the studio's spatial resources, the project's high precision expectations left its place to personal experimentations with the accessible resources. While reformulating the project with accessibility in mind, "Water" stood out as the most accessible and controversial fundamental resource. It is introduced to the studio as an accessible, political, physical, chemical, and biological entity. Hydrophilia project focuses on water as a "resource" in this context and developed the material research, narrative, and formation processes through it. The research continued with the site selections based on the personal researches of each student. It is aimed to design a structure using both water experiments and findings obtained from the site research. For example, a student investigates the evaporationsublimation dynamics of water with experiments at home (Fig.6). The next stage is documenting formations using drawings and models (Fig.7). During the research phases on water, experiments with accessible and diverse materials were encouraged. 


\section{Journal of}

Design Studio

v:3 n:1 July 2021
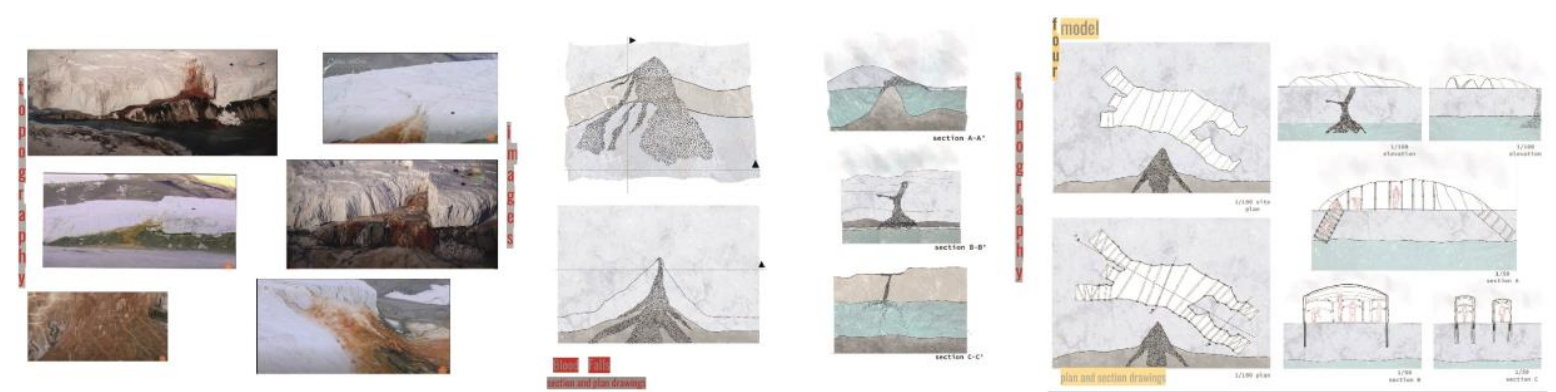

Figure 7: From left to right: Online research, site drawing and process, Research Center design. Project: Pinar Zeyrek

The third adaptation was introducing additional mediums to enrich studio productions with interactive collaboration and learning experience. In addition to the regular studio meetings on Zoom with student-instructor interactions, student-student, student-studio interactions in different mediums such as Zoom rooms, Mural, Miro, Google Slides are proposed to replace exhibition, collaboration, and discussion sessions. As a part of this diversity, logbooks also played a key role in creating continuous student interaction within the studio. Furthermore, since the simulation of the process-based nature of the studio through Zoom or whiteboards is impossible, a new medium has been proposed to reinforce the interaction. As a result, it was experienced that sharing the daily productions and jury presentations with the whole studio decreased the isolation of the students in the process. Moreover, meeting with different groups of students and working together in workshops contribute to the sense of community.

\section{Site}

Second-term projects were generally related to the research and experience of the site. Therefore the inaccessibility of the site with pandemic restrictions caused significant changes in the studio. Before COVID-19, students were expected to transform "site" information into project data using diverse methods. The dynamics of the site ranged from the dense urban area to the rural fields, and the data extracted from them formed cornerstones of the projects. Observation of the theoretical knowledge introduced by the project instructors on-site expanded the spatial layers of the atmosphere, function, and daily life. When students step outside of studio space and activate the studio setup, they start to learn from the space itself by physical documentation such as photography and measurements, lectures from the local experts or governors, interviews with users to learn the informal history of the site. Students were encouraged to collect all the data from the site and filter it with the given project descriptions.

Contextual and material expectations of the precovid studio are revised from collective experiences to individual ones in order to recreate an accessible studio theme. Reevaluation of the resources manifests itself with a new "familiar" and "accessible" approach to the site for including the site experiences and its information. In order to discuss changing expectations of the studio, pre-covid projects might be analyzed in comparison: The first project, "Speculative Landscapes" had focused on forming an imaginary landscape in different scales and analysis over time. The project was held by experimental models constructed with material researches and their conceptual meanings by prioritizing the observation. The second was a structure project aiming to translate knowledge of the experienced site into an architectural realm with a detailed architectural project by discussing it with tectonic aspects. Adaptations had been proposed in order to preserve observation and experience of the project sites.

The first adaptation aims to perceive the site as a "place" more than a location for analyzing it from a distance. Hydrophilia discusses the site 


\section{Journal of \\ Design Studio \\ v:3 n:1 July 2021}

with inputs from online resources. The project is started with online research regarding the chemical, biological, physical, and political properties of water, followed by experiments. Observation-based research is carried to a tangible level with experiments. Through material studies, students were able to experiment from their homes with the familiar material of water and have the chance to observe tectonic formations with various interventions (Fig.8). In the first step, students were encouraged to choose the material properties forming the site instead of a specific location. By doing so, the site is freed from location-based data and transformed into a material entity.
The next step is research and observation of the project site to analyze the water formations throughout time. The site is analyzed from different resources such as maps, videos, and research papers. Then, they are used as inputs for the projects; sequential drawings are used to analyze the transformation process of the site and water features (Fig.9). The initial project is not only a well-functioning result of the research process but also a systematic structure design (Fig.10). Remote analysis of the site precluded detailed analysis and limited the detail level with accessible maps and photographs. Therefore structural principles and site-specific adaptations of the project could not be discussed as detailed as pre-covid terms. On the other hand, the holistic approach
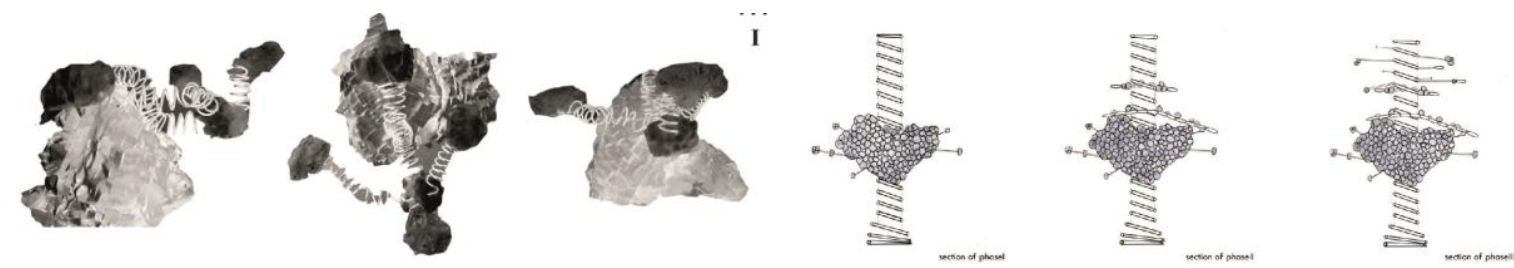

Figure 8: Phase-model of water experiment and phase-drawing of water experiment. Project: Sude Daban
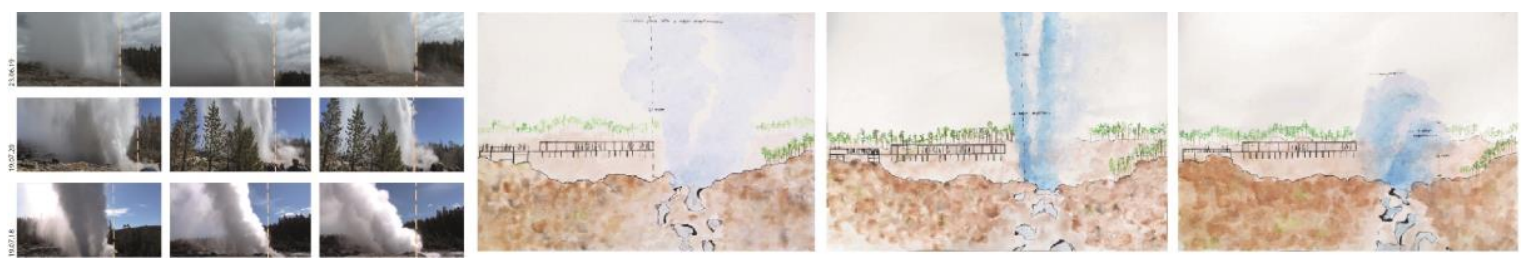

Figure 9: Temporal analysis of the geyser from videos, and design proposal according to the changes of geyser. Project: Zeynep Zehra Avcı
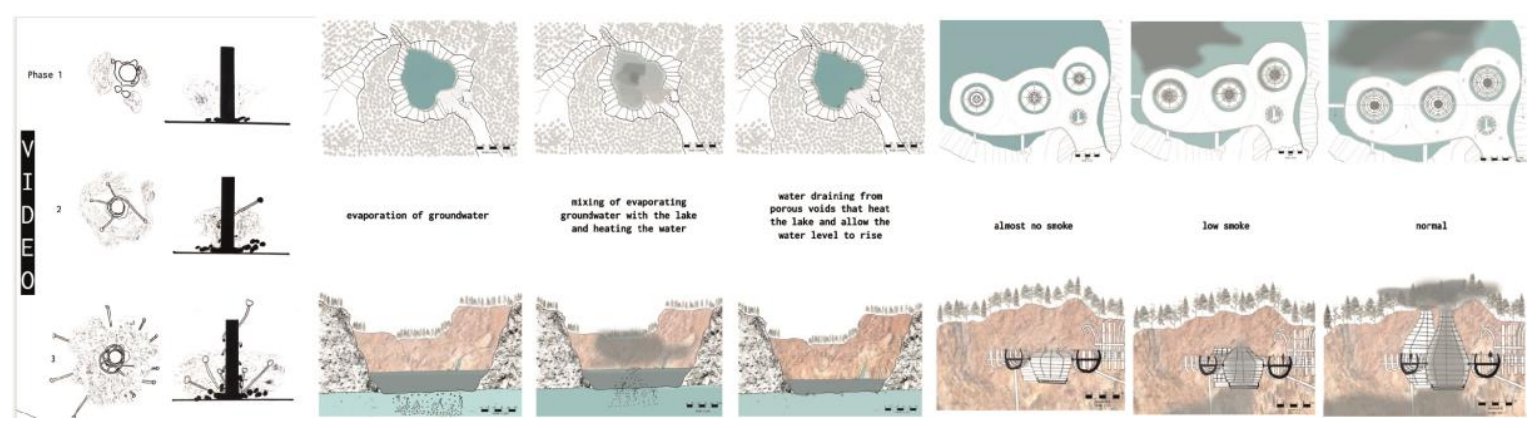

Figure 10: From left to right: Water-based experiment diagrams of formation in 3 phases, Site formation in 3 phases, Design idea formation in 3 phases. Student: Seyid Ömer Yă̆murcu 


\section{Journal of \\ Design Studio \\ v:3 n:1 July 2021}

of the project meeting material and structural experiments on the same project and personalization potential offers alternative approaches to the lacking context. Students were able to design a structure discussing complex spatial relationships from the accessible information and documents.

The second project, Perform-X, focuses on developing complex spatial relationships. Students are expected to design a performance space to an accessible site at a maximum 10 minutes distance by walking to their houses to replace information given by instructors with extensive knowledge of each student. The diversity of the sites and contexts uncovers various spatial and contextual relationships from rural to metropolitan areas. The project process continues similar to previous terms: students create mappings of the surrounding area where they are familiar and then interpret the theme by considering the site's unique social and historical context. Then it continued with collecting information about the site from accessible resources and their experiences and followed with the definition of the programmatic requirements of the site (Fig.11).
Accessible resources theme helped students to collect information from their own accessible sites with direct experiences; which is related with the project theme since it is subjected to the "frequented" places. Students are expected to discuss the changing usage of the area before and after lock-down. Inhabitants of the surrounding area become an important input like the students themselves. Site is analysed at a material level with both objective physical information acquired from the local authorities and also their physical experiences then transformed all into a site model (Fig.12).

While this paper is written, Perform- $\mathrm{X}$ is still an ongoing project. To compare the existing studio outcomes before and after COVID-19; even though the diversity of the sites produced a rich discussion environment in the studio, it was not possible to compare the different approaches to the site and detail it with other research in the studio. Moreover, familiar and accessible themes made the detailed reading and analysis of each site possible with personal filters of the students and enabled the studio to construct architectural designs on the current states of the site.
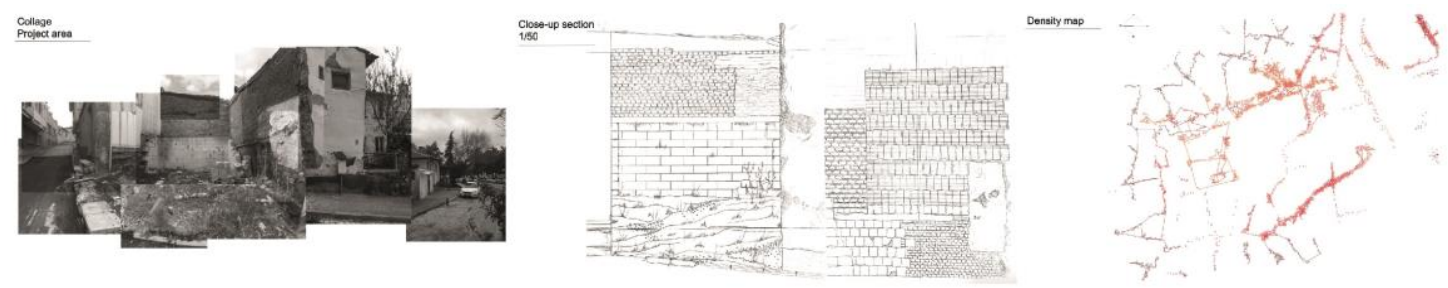

Figure 11: Site collage, site survey drawings and mapping on the site. Student: Dilay Yücaltı
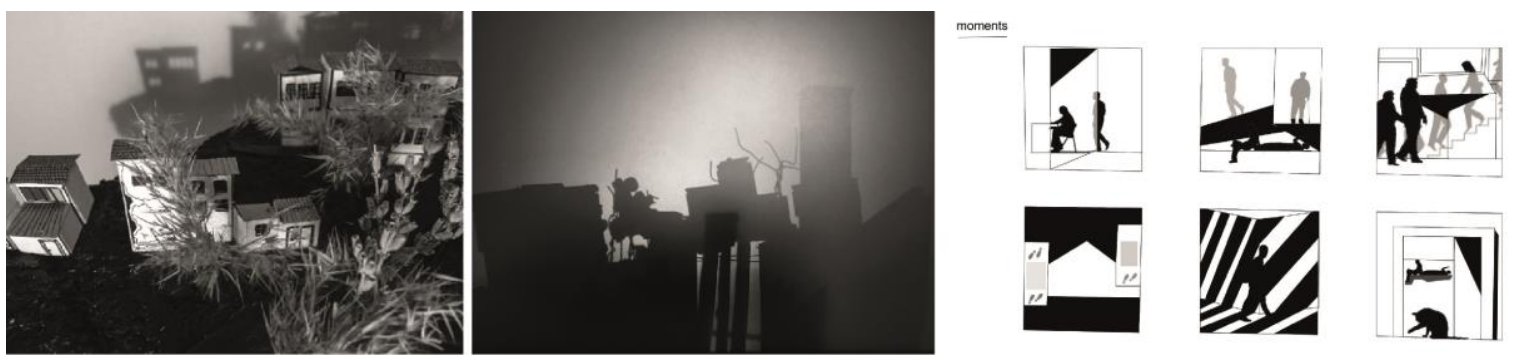

Figure 12: Site model, analysis through the site model photographs and 'Performance' program through sequences of shadow and movement. Student: Dilay Yücaltı 


\section{Journal of \\ Design Studio}

v:3 n:1 July 2021

\section{Conclusion}

Introducing new resources and adaptations to the remote studio are detailed in three categories; curriculum, studio as a workspace, and site. While all the adaptations aim to create the studio environment in virtual mediums by reassessing the tools and resources through accessibility, it also enables working alone yet together. Accessible resources shift the instructor's role from 'source of the knowledge' to 'facilitator' collecting and integrating the knowledge to the studio (Yorgancioğlu, 2020). The instructor acts as the curator of the studio by collecting, organizing, and exhibiting the individual processes.

The first adaptation under the curriculum is including the new resources "Wednesday Workshops" and "Logbooks" which make the curriculum live, visible and shareable. The outputs revealed the significance of these intermediaries for both students and instructors to track the process. Additionally, collections of analog productions that are the primary medium for first-year students were always problematic during regular terms. Due to the inevitable documentation for communication and shared ground with logbooks, an extensive collection is created more comprehensively than ever. As a result, introducing all the natural encounters deliberately to the term program with workshops, diverse media, and mediums leads to implementing a more structured and instructor-led curriculum. Even though activating students is the primary goal, artificially creating all the enriching factors increased the share of the instructor in the studio.

Another adaptation was the reconstruction of studio structure on online platforms. The paired instructor and group system proposed as an alternative for the spotlighted-authoritative nature of online education to form equal ground and atmosphere of the design studio. Furthermore, replacing the traditional materials and techniques of the first-year studio with accessible ones enriched studio discussions. Also, their discussion and exhibition invited more channels of communication such as Mural, Miro, Google Slides in addition to the usual Zoom environment to create a productive studio atmosphere. However, despite the increasing diversity of the discussions, the precision of individual projects remained lower than regular term expectations.

The last adaptation was related to the site. The collective observations about the site like survey and experience are needed to be replaced with accessible or familiar ones. The locational richness of the site is aimed to be introduced from different perspectives in two projects. The site's physical and material properties play a crucial role in defining specific processes of the projects through analysis of water elements in Hydrophilia project. In order to highlight the social characteristics of the site, students focused on the closest and familiar sites to their house and developed their design based on personal observations through the Perform-X project. Remote analysis of the site, precluded detailed analysis and limited the detail level with accessible maps and photographs. Students were able to design a structure discussing complex spatial relationships from the accessible information and documents. Therefore structural principles and working mechanisms of the project could not be discussed as detailed as pre-covid terms. Nevertheless, the fragmented structure of the projects enabled students to handle projects in different material and structural approaches.

\section{Acknowledgements:}

MEF FADA First Year Architectural Design Studio 2021 Spring Term tutors Başak Eren, Beril Sarısakal, Derya Uzal, Didem Sağlam, İrem Korkmaz, Naz Kaya, Zelal Rahmanalı Nottrott, Zeynep Bacınoğlu for their contribution to the formation and execution of the curriculum throughout the years, all students for their works and invited lecturers for their support.

\section{References:}

Corazzo, J. (2019). Materialising the Studio. A systematic review of the role of the material space of the studio in Art, Design and Architecture Education, The Design Journal, 22:sup 1, 1249-1265,

DOI: $10.1080 / 14606925.2019 .1594953$ 
Schön, D. (1987). Educating the Reflective Practitioner: Toward a New Design for Teaching and Learning in the Professions, San Francisco, CA: Jossey-Bass

Yorgancioglu, D., (2020). Critical Reflections on the Surface, Pedagogical and Epistemological Features of the Design Studio under the "New Normal" Conditions, Journal of Design Studio, V.2, N.1, pp 25-36 